Article

\title{
Application of Ultrasonic Atomization in a Combined Circulation System of Spray Evaporative Cooling and Air Cooling for Electric Machines
}

\author{
Yu Wang ${ }^{1,2} \mathbb{D}$ and Lin Ruan $1,2, * \mathbb{D}$ \\ 1 Institute of Electrical Engineering (IEE) of Chinese Academy of Sciences (CAS), Beijing 100190, China; \\ wangyu@mail.iee.ac.cn \\ 2 University of Chinese Academy of Sciences, Beijing 100049, China \\ * Correspondence: rosaline@mail.iee.ac.cn
}

Citation: Wang, Y.; Ruan, L. Application of Ultrasonic

Atomization in a Combined Circulation System of Spray Evaporative Cooling and Air Cooling for Electric Machines. Processes 2021, 9, 1773. https://doi.org/10.3390/ pr9101773

Academic Editor: Myung-Seop Lim

Received: 30 August 2021

Accepted: 28 September 2021

Published: 2 October 2021

Publisher's Note: MDPI stays neutral with regard to jurisdictional claims in published maps and institutional affiliations.

Copyright: (c) 2021 by the authors. Licensee MDPI, Basel, Switzerland. This article is an open access article distributed under the terms and conditions of the Creative Commons Attribution (CC BY) license (https:// creativecommons.org/licenses/by/ $4.0 /)$.

\begin{abstract}
A combined circulation system of spray evaporative cooling and air cooling (CCSSECAC) is a way to enhance the cooling performance of an air-cooled electric machine while maintaining its existing structure. Based on a traditional air-cooled machine, when the discrete evaporative cooling medium particles are scattered into the airflow, they will reach the heat source with the air circulation. The cooling capacity of the cooling system is enhanced simultaneously through the phase transition and convection heat transfer. Ultrasonic atomization is a simple way to produce tiny droplets and a good way to improve the performance of CCSSECAC. To verify the effectiveness of such a system, a principle test model was built and a multi-operational condition experiment was carried out as an exploratory study. The experimental results showed that the new cooling system was feasible for horizontal machines, and the stator coil temperature was significantly reduced compared with the air-cooled mode.
\end{abstract}

Keywords: electric machines; spray; evaporative cooling; air cooling; ultrasonic atomization; airflow friction loss

\section{Introduction}

As is well known, the larger the capacity of the single electric machine, the less material consumption per unit capacity and the more economical it is. Increasing the linear size and increasing the electromagnetic parameters are two main ways to achieve these goals. No matter which technical route is adopted, the growth rate of the heat dissipation area cannot catch up with the growth rate of the loss. An air-cooling system is the earliest cooling technology for electric machines. Its simple structure, convenient maintenance, safety and reliability were welcomed by manufacturers and consumers. However, there is no denying that, restricted by the physical properties of air, the capacity of the air-cooled machine increases more and more slowly. Both hydrogen cooling and water cooling are good alternatives to air cooling, but they have their limitations, such as the risk of explosion and short circuits.

Spray cooling of electric machines was attempted 100 years ago. In recent years, with the development of electric vehicles, oil spray cooling has been applied to drive motors with a high power density [1-3]. Liu et al. systematically tested the performance of an oil-cooling system using three types of commercial spray nozzles and gave design suggestions [4]. Oil is chosen as a cooling medium mainly because of its insulation.

Freon is also a good dielectric fluid. At the beginning of this century, this evaporative cooling technology, which has been studied for more than 60 years at the Institute of Electrical Engineering, Chinese Academy of Sciences (IEECAS), was successfully applied in a giant hydrogenator at the Three Gorges Hydropower Station. Around the same time, the spray evaporative cooling technology was invented and used for high power density motor 
cooling to realize the existence of a high current density and low winding temperature [5]. However, only the end windings are cooled by spray in that motor. Tao Yu et al. introduced the material used in Freon spray to cool a medium-voltage asynchronous motor [6].

The application of spray cooling in motors mainly depends on spraying droplets directly to the end of the winding, which then realizes high-efficiency heat dissipation through convection and phase transformation. This series of processes includes atomization, jet cooling and evaporative cooling. In terms of atomization research, Liu ShaoYan et al. used PDPA to measure the spray parameters of the nozzle [7]. In terms of jet cooling, Haixiang Zhang et al. paid attention to the effect of surface wettability on water droplet spreading and splashing [8]. Tian, J. and Chen, B compared the different effects of droplet diameter, impact velocity, viscosity and surface tension on the impaction [9]. In terms of evaporative cooling, Zang [10], Sazhin [11], Volkov [12] and Strizhak [13] did a large amount of work on the dynamic model.

Although direct spray cooling has a high heat transfer coefficient, there are two weaknesses that spray cooling must overcome [14]. One is the requirement of a large pressure drop to produce fine droplets. The other one is the risk of plugging fine nozzle holes. A large pressure drop means that complex pumping equipment is needed to keep the system running. The possibility of clogging may lead to surface burnout.

In an electric machine cooling system, spray cooling also faces the problem that other electronic cooling devices do not need to consider, that is, how to let the droplet travel far enough with the airflow and through the complex structure inside the motor to reach the surface of each heat source. Therefore, the spray cooling techniques that were successfully applied, especially the non-phase change cooling techniques, such as oil spray cooling, only spray the working fluid to the end of the winding and do nothing to the effective part of the winding [4]. Haihong Dong et al. investigated an air/spray cooling system with Freon for turbine generators and noted that the phase change process can greatly enhance the cooling effect compared with air cooling [15]. However, as described above, the system must be equipped with high-pressure circulating pumps and filters.

Ultrasonic atomization avoids the shortfalls of spray cooling. It is simple in structure, easy to control, does not need a high-pressure pump for liquid supply and is very easy to install according to the needs of the project. The droplets are so small that they can be carried well by the airflow.

When the source vibrates at a frequency greater than $20 \mathrm{kHz}$, the surrounding liquid may be crushed into small droplets. We call this ultrasonic atomization. The formula summarized by Robert J. Lang based on experiments is still used to calculate the diameter of droplets that are generated by ultrasonic atomization [16]:

$$
D=0.34\left(\frac{8 \pi \sigma}{\rho f^{2}}\right)^{1 / 3}
$$

where $D$ is the average droplet diameter $(\mathrm{m}), \sigma$ is the surface tension coefficient $(\mathrm{N} / \mathrm{m}), \rho$ is the liquid density $\left(\mathrm{kg} / \mathrm{m}^{3}\right)$ and $f$ is the ultrasonic frequency $(\mathrm{Hz})$.

Later, scholars continued to improve the ultrasonic atomization model. The effects of liquid phase properties on ultrasonic atomization were studied by Avvaru et al. [17]. Zhang et al. examined the effects of the operating parameters based on the capillary wave and cavitation hypotheses and derived a correlation equation to predict droplet sizes [18]. High-speed imaging was employed by Deepu to measure the average atomization lifetime of the droplet. Nevertheless, Equation (1) is still widely used.

\section{Introduction of a Combined Circulation System of Spray Evaporative Cooling and Air Cooling for an Electrical Machine}

The structure of a combined circulation system of spray evaporative cooling and air cooling (CCSSECAC) for an electrical machine is an optimization method for improving a traditional air-cooled machine. In this system, the right amount of discrete evaporative cooling medium particles, i.e., Freon, is mixed with the airflow to participate in the air 
circulation. The refrigerant absorbs heat and, once it reaches its boiling point, vaporizes and absorbs much more heat. Figure 1 is a schematic of CCSSECAC. The nozzles or atomizers are arranged downstream of the fan. The mist evaporative cooling medium enters into the stator and rotor with the air circulation. Some of the droplets attach to the heat source surface for heat absorption and gasification. Some of the droplets are directly heated and undergo gasification by hot air to reduce the temperature of the cooling air. The gaseous coolant is condensed into a liquid as it flows through the condenser with air. The liquid refrigerant is collected into a tank for subsequent circulation. As the liquid medium almost does not pass through the fan, it avoids the liquid loss and friction loss caused by the attachment of droplets to the fan.

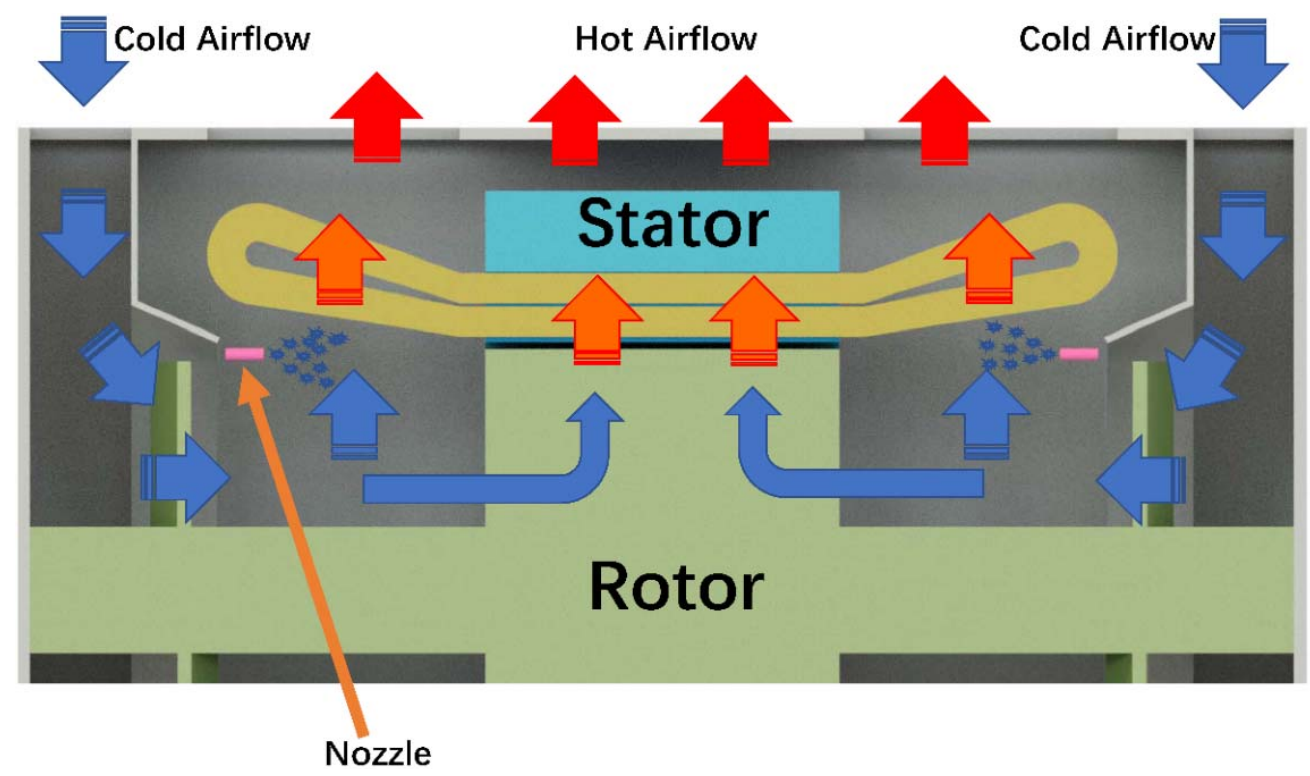

Figure 1. The schematic of the combined circulation system of spray evaporative cooling and air cooling (CCSSECAC).

Due to the narrow and complex airflow channel inside the motor, it is required that the movement state of Freon droplets is highly like that of the airflow.

A droplet with good followability should be small in diameter and density. When the density of a droplet is much higher than that of the gas, the velocity of the droplet can be calculated using the following formula [19]:

$$
\begin{aligned}
U_{p}(t) & =U\left[1-e^{-\frac{t}{\tau_{s}}}\right] \\
\tau_{s} & =d_{p}^{2} \frac{\rho_{P}}{19 \mu}
\end{aligned}
$$

where $U$ is the gas velocity, $\mu$ is the dynamic viscosity, $d_{p}$ is the droplet diameter and $\rho_{P}$ is the droplet density.

Obviously, the droplet diameter has a great influence on the followability. The atomizing nozzles, both two-fluid nozzles and single-fluid nozzles, need high pressure and a very small aperture to form tiny droplets. In contrast, creating small droplets by an ultrasonic atomizer is easy. The droplets of R113, with no need to pressurize the fluid, can be less than $2 \mu \mathrm{m}$ with an ultrasonic nebulizer. Based on the above reasons, we decided to use an ultrasonic atomizer as the atomization device for CCSSECAC.

\section{Design of the Experiment}

An experimental platform was used to verify the feasibility of an ultrasonic atomization device for CCSSECAC. Figure 2 is the schematic diagram. The platform was composed of two parts: the airflow path and the liquid path. The airflow path, identified by the red 
arrows, is the channel where air and coolant participate in a heat exchange cycle. The liquid path, represented by blue lines, is a set of pipe fittings that are specially used for the coolant in a liquid state. One function of the liquid path is to collect the overflow and condensate coolant. Another function of the liquid path is to supply the liquid for the ultrasonic atomizer.
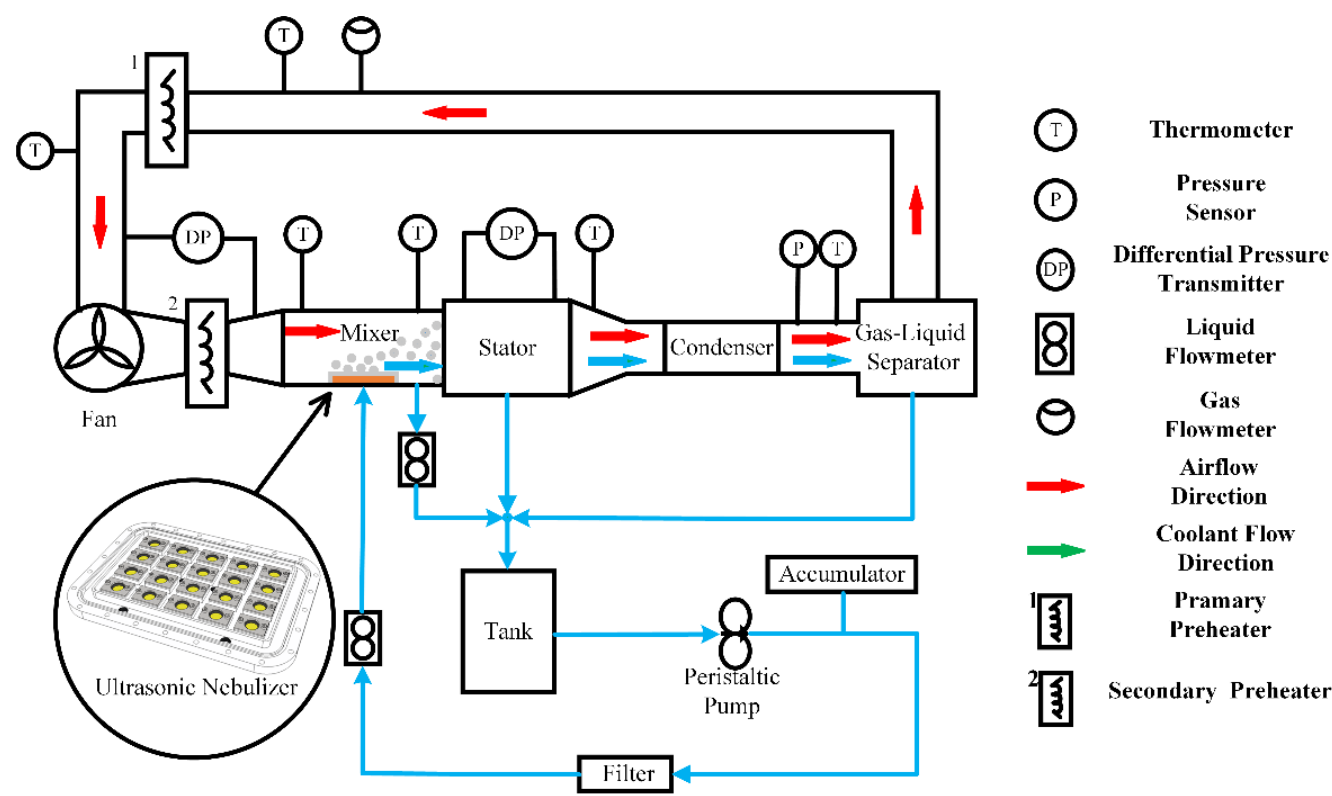

Figure 2. The schematic diagram of the experimental platform.

The intersections of the two paths involve the mixer, the stator model, the condenser and the gas-liquid separator. Beyond them, each piece of auxiliary equipment is successively connected in series to form a set of closed-loop airflow conduits. The auxiliary equipment of the airflow path includes return ducts, preheaters and a fan. The purpose of installing two preheaters near the fan is to accurately control the air temperature when it goes into the stator model. The air that is blown out by the fan is fully mixed with the coolant droplets that are produced by ultrasonic atomization in the mixer and then enters the stator model. In the stator model, the droplets absorb heat and are gasified while following the airflow through the ducts of the stator. After the hot air reaches the condenser, it is cooled rapidly. Most of the coolant condenses, collects, and eventually flows back into the tank. The remaining coolant steam flows to the fan with air in a saturated state.

The coolant in the liquid storage tank is driven by a peristaltic pump to the ultrasonic atomizer, where most of it is atomized and flows away with the airflow. To prevent the ultrasonic nebulizer from running in a liquid-deficient regime, it is supplied with an excess of liquid, which will return to the tank from the overflow hole. By recording the difference between the inlet and outlet flows of the ultrasonic atomizer, the actual flow rate of coolant involved in heat transfer with the air can be obtained. The ultrasonic atomizer used in this study consisted of an array of 20 ultrasonic oscillators with a diameter of $17 \mathrm{~mm}$ and a frequency of $1.7 \mathrm{MHz}$. Because the flow provided by the 10 oscillators made the windings colder than $90{ }^{\circ} \mathrm{C}$ at the maximum thermal load, no more oscillators were used in the experiments.

The core components of the experimental platform were the stator model and the mixer. As shown in Figure 3, the stator model was modeled as part of the stator of a real high-voltage motor. The main dimensions were copied 1:1. In the stator model, there were seven slots and four ventilation ditches altogether. Like a true motor with double-layer winding, there were two stator coil bars in each slot; therefore, the common model had a total of 14 bars. A mixer was arranged at the front end of the stator model, where there is a rotor in a real motor. An ultrasonic atomizing device was installed at the bottom of the mixer to mix the air with the atomized coolant. 


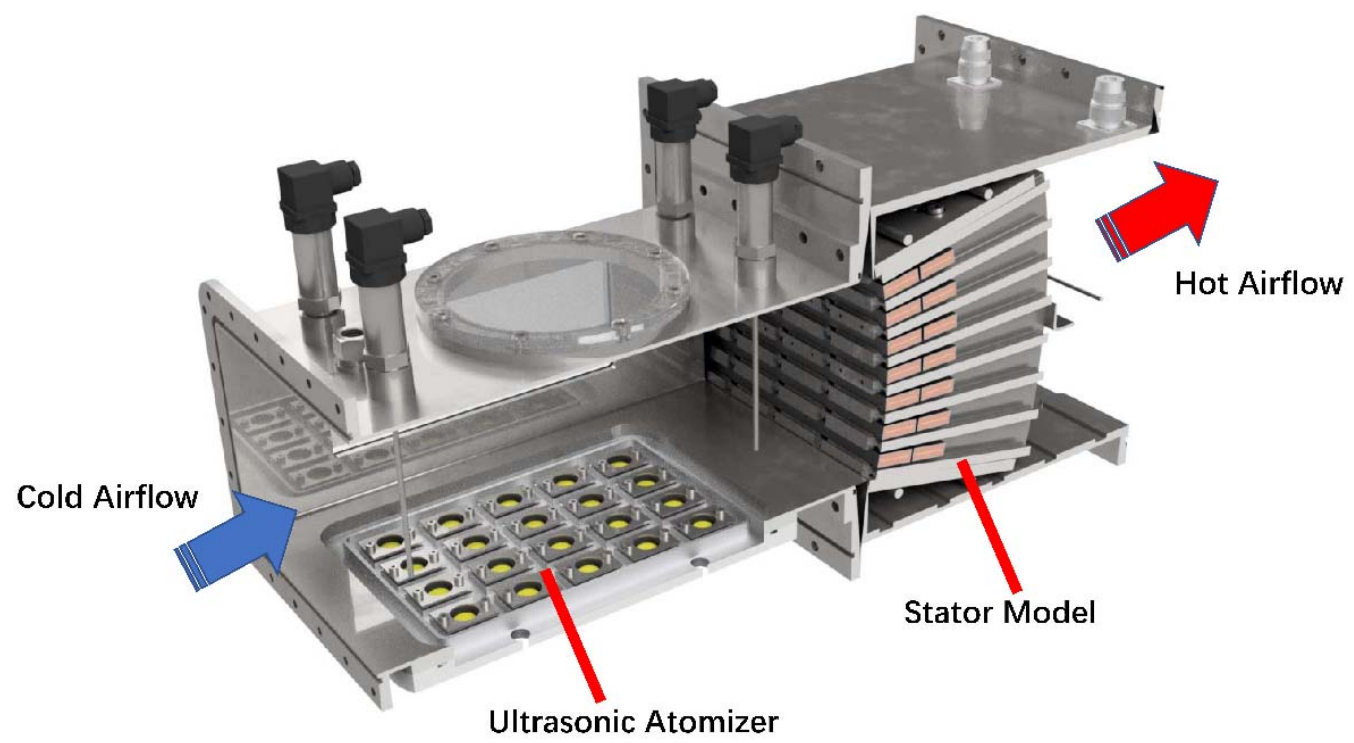

Figure 3. The rendering of the mixer and stator model.

The only difference between the stator model and the real machine was the design of the heat source. To avoid the influence of the heat generated in the end winding on the experimental results, the coil bars were designed based on the volumetric heat flux of the real winding. One coil bar was composed of two copper bars, holding a thin-film heater, and the main insulation. The main insulation thickness, volumetric heat flux and outside dimensions of the coil bars were equal to those of the prototype machine. Thin-film heaters were arranged in the teeth and yoke of the core with the purpose of simulating the influence of the core loss distribution. Therefore, the copper loss, tooth iron loss and yoke iron loss could be controlled separately in the stator model.

As shown in Figure 4, several T-type thermocouples were arranged in slots. All the even-numbered thermocouples were arranged in the positions corresponding to the ducts, and the odd-numbered thermocouples were arranged in the opposite positions. The thermocouples numbered (1) and (2), (7) and (8) and (13) and (14) were, respectively, arranged in the bottom, middle and top strips, that is, outside the main insulation. These thermocouples were arranged in all the slots. In the middle one of the seven slots, thermocouples were also placed inside the main insulation. The thermocouples numbered (3), (4), (5), (6), (9), (10), (11) and (12) were located inside the main insulation, corresponding to the thermocouples in the strips. They were used to measure the temperature gradient at the insulation.

In the middle slot, two additional thermocouples were arranged separately inside the insulation and at the long side of the bars, that is, near the tooth. The two thermocouples are represented in Figure 5 and labeled as (15) and (16). The two points are placed here because we expected them to be hotter than the others.

As mentioned earlier, the stator model used an electrothermal thin film to simulate copper loss in the windings. The relationship between the electric heating power and the equivalent current density of the analog windings is listed in Table 1. During the experiment, the heating power was adjusted according to this relation. In the later part of this paper, the electrical heating power is directly expressed using the current density. As the iron loss is only slightly affected by load changes under fixed-frequency conditions [20], the iron loss was set to $150 \mathrm{~W} / \mathrm{Kg}$ in this experiment, equivalent to $3 \mathrm{~W} / \mathrm{Kg}$. 


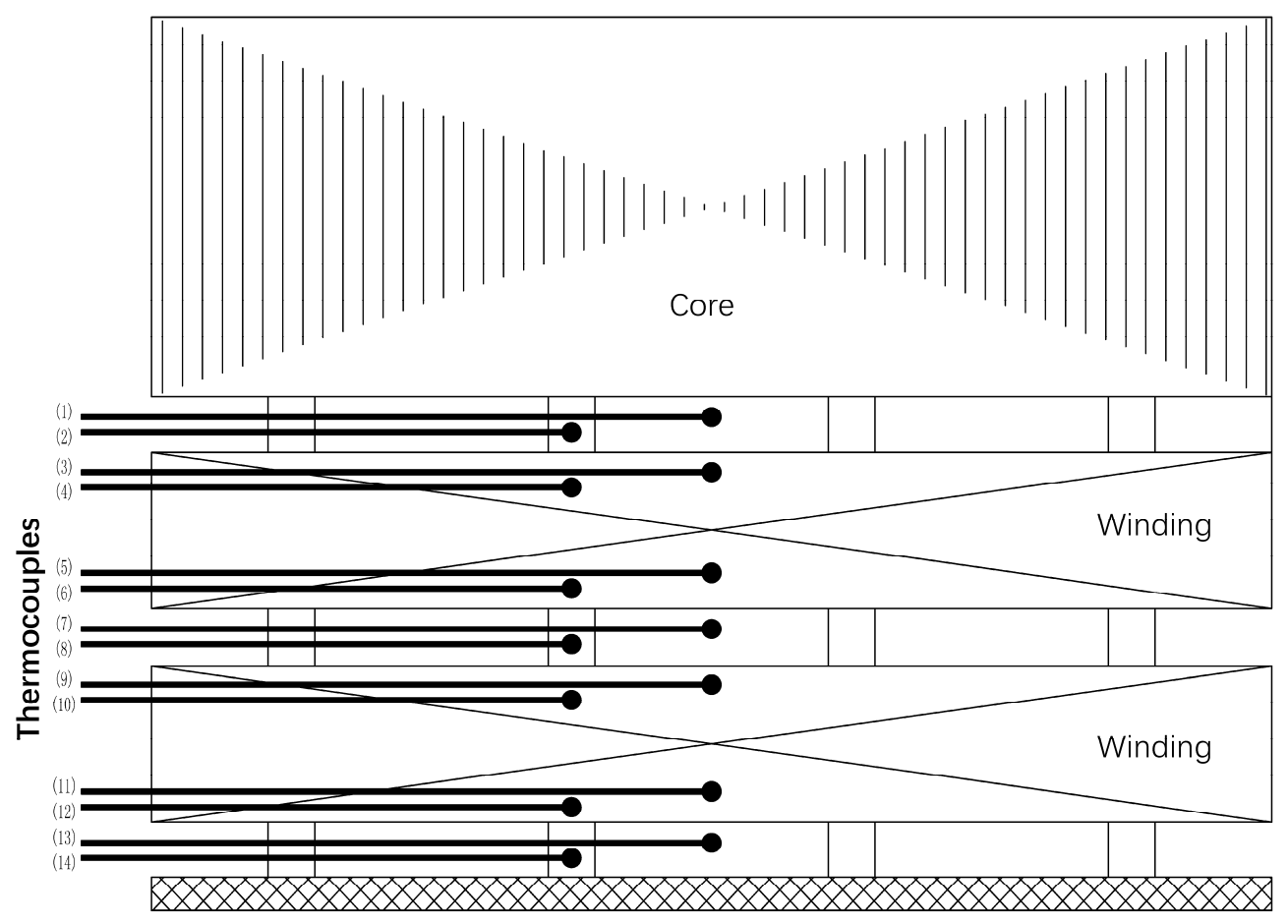

Figure 4. Thermocouple arrangement in an axial section (not drawn to scale).

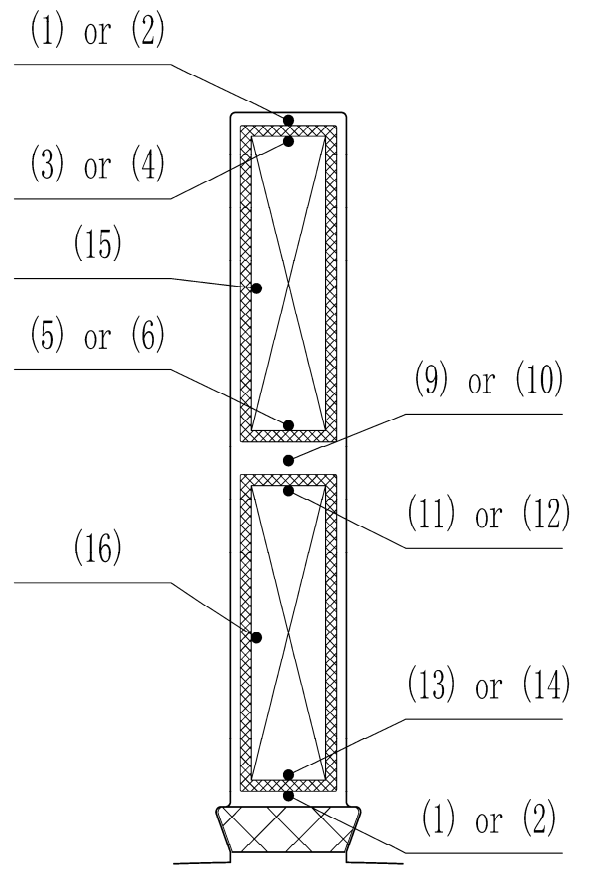

Figure 5. Thermocouple arrangement in a radial section (not drawn to scale).

Table 1. The conversion relationship between the equivalent current density and heating power.

\begin{tabular}{ccccc}
\hline Heating Power $(\mathrm{W})$ & 180 & 319 & 499 & 718 \\
\hline Equivalent Current Density $\left(\mathrm{A} / \mathrm{mm}^{2}\right)$ & 3 & 4 & 5 & 6 \\
\hline
\end{tabular}

In this project, traditional air cooling and CCSSECAC were operated successively. The air-cooling experiments were first completed to prevent the coolant from volatilizing into the air and affecting the physical properties of the circulating airflow. In addition to the 
rated working condition, the main variables that were controlled during the experiment were temperature rise and airflow speed. The airflow speed that was controlled in the experiments was not the airflow speed in the ducts of the stator, but rather the value measured by the gas flowmeter in the return duct. Therefore, this means that we compared the relative volume flow rate of air in this project. For all working conditions, the ambient temperature was defined as the air temperature into the stator, which was controlled at $40 \pm 0.5^{\circ} \mathrm{C}$ according to the IEC60034-1 standard. Each working condition was repeated at least three times.

When designing a cooling system, we may not only ensure that the temperature rise does not exceed the design limit but also take efficiency into account. Therefore, while considering the enhanced heat transfer effect of spray cooling, its influence on the airflow friction loss should not be ignored. In the experiments, the pressure drops that were generated by the air flowing through the stator model were measured as the scale of airflow friction loss.

The airflow speed was set to 0.6, 0.7, 0.8, 1.0, 1.5 and $2 \mathrm{~m} / \mathrm{s}$. Because the airflow friction loss of CCSSECAC increased very fast with increasing airflow speed, it became unacceptable at an airflow speed of $2 \mathrm{~m} / \mathrm{s}$. Therefore, the airflow speed of $2 \mathrm{~m} / \mathrm{s}$ was only used in the air-cooling test.

The main measuring equipment used in the experiment were T-type thermocouples and a differential pressure transmitter. A Fluke 2680 Data Acquisition System was used to record the data. T-type thermocouples that were calibrated by Fluke 9170 Metrology Well were used in the experiments to achieve a measurement accuracy of $0.5^{\circ} \mathrm{C}$. The range of the differential pressure transmitter was $0 \sim 250 \mathrm{~Pa}$ and the accuracy was $0.1 \%$.

\section{Results}

\subsection{The Optimal Temperature Measurement Position for Temperature Rise Experiments}

No doubt the higher temperature rise measurements will be detected by thermocouples located in the main insulation. Because they are in direct contact with copper. However, we still need to know where the temperature measuring points are arranged to more accurately reflect the maximum temperature during experiments.

Figure 6 compares the values from six temperature measuring points placed inside the main insulation. In the figure, the arrangement sequence of the temperature measurement points from bottom to top in the slot is shown in the sequence from left to right. Temperature values were collected in the two working conditions of the maximum load of the air-cooling experiment and the maximum load of the CCSSECAC experiment. It can be seen that when the two temperature measuring points, namely, no. (15) and no. (16), were not considered, the temperature measured values rose successively from the bottom to the top of the slot. However, the temperature difference between these four points was not large. The temperature measured at the two points on the long side of the coil bar section was $3-5{ }^{\circ} \mathrm{C}$ higher than the above four points. The measurement of thermocouple (16) was therefore used to calculate the temperature rise.

In engineering applications, it is not possible to embed the temperature-measuring device inside the main insulation of the coil. The side of the coil does not have enough room for temperature probe mounting because it is close to the teeth. Therefore, the temperature-measuring element is usually installed in the strip. Figure 7 quantitatively compares the temperature gradients of the main insulation at the contact position between the main insulation and the strips. Each curve in the figure was derived from the difference between the temperature inside the insulation and the temperature outside the insulation at a location. The definitions of the curves are listed in Table 2. All data were collected when the temperature rise measured by thermocouple No. (16) was equal to $50{ }^{\circ} \mathrm{C}$. 


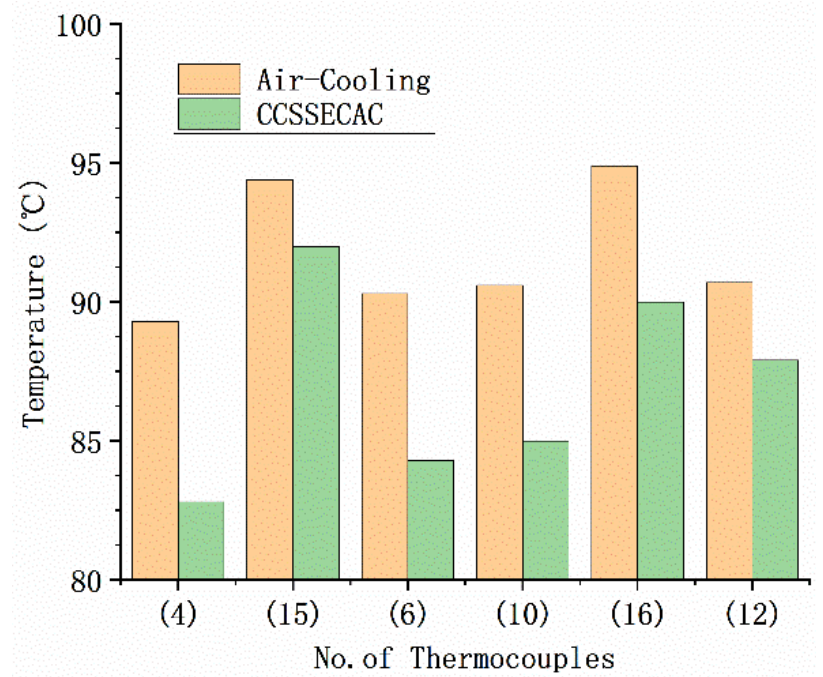

Figure 6. The temperatures at different positions.

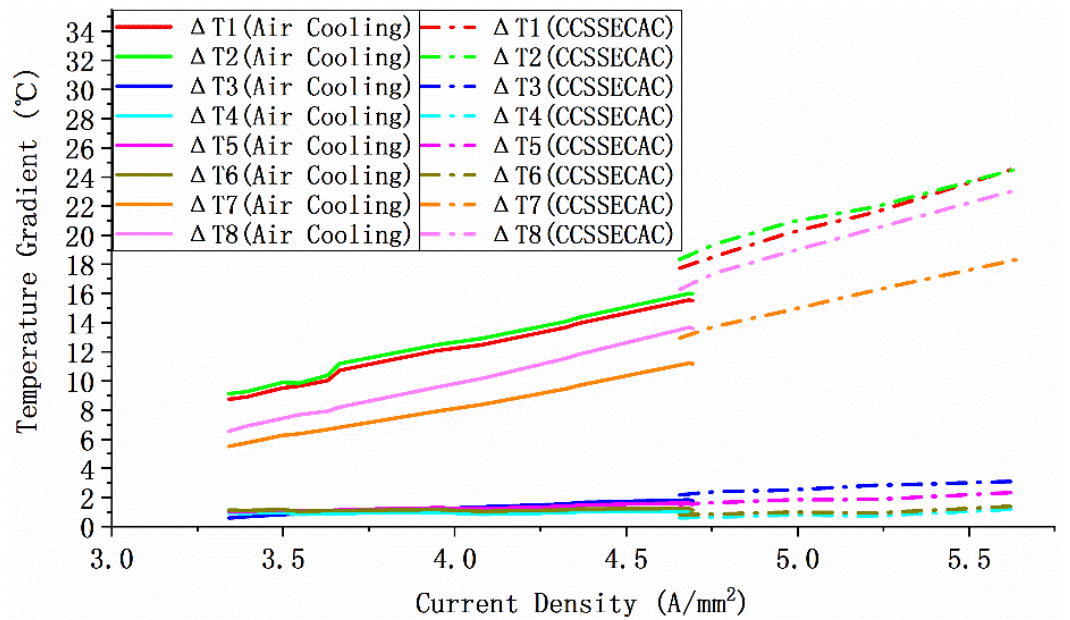

Figure 7. The temperature gradients at different positions.

Table 2. Definitions of the labels of the curves in Figure 7.

\begin{tabular}{cc}
\hline Label & Definition \\
\hline$\Delta \mathrm{T} 1$ & Difference between thermocouples (14) and (12) \\
$\Delta \mathrm{T} 2$ & Difference between thermocouples (13) and (11) \\
$\Delta \mathrm{T} 3$ & Difference between thermocouples (10) and (8) \\
$\Delta \mathrm{T} 4$ & Difference between thermocouples (9) and (7) \\
$\Delta \mathrm{T} 5$ & Difference between thermocouples (6) and (8) \\
$\Delta \mathrm{T} 6$ & Difference between thermocouples (5) and (7) \\
$\Delta \mathrm{T} 7$ & Difference between thermocouples (4) and (5) \\
$\Delta \mathrm{T} 8$ & Difference between thermocouples (3) and (1) \\
\hline
\end{tabular}

It is obvious from the figure that the measured temperatures of the thermocouples that were placed in the bottom and top strips were much lower than the temperature of the copper. The temperature gradient of the main insulation next to the middle strip was very small. Further, because of the better heat dissipation conditions, it was easy to measure a relatively low value using the thermocouples located in the position corresponding to the ducts. Therefore, the temperature sensor in the stator slot is recommended to be installed in the middle strips and kept away from the ducts. 


\subsection{Comparison of the Cooling Effects under Low Load Conditions}

The rated working condition was the minimum heat load in this project. When the coil heating power was $200 \mathrm{~W}$, the equivalent electric density was $3.17 \mathrm{~A} / \mathrm{mm}^{2}$. The electric density of air-cooled motors is usually set between 3 and $3.5 \mathrm{~A} / \mathrm{mm}^{2}$. To keep things simple, the rated working condition was set to $3.17 \mathrm{~A} / \mathrm{mm}^{2}$.

Figure 8 compares the temperature rise and airflow friction loss of the two cooling systems at different airflow speeds under the rated working conditions. The airflow friction loss here was represented by the pressure drops at the stator model. The results show that the temperature rise of the winding could be greatly reduced by CCSSECAC at a low load level. Especially in a small airflow speed range, the temperature rise was reduced by half. With the increase in airflow speed, although the temperature rise of CCSSECAC was still much lower than that of air cooling, its pressure drops increased much faster. The temperature increase of the air-cooling system at an airflow speed of $2 \mathrm{~m} / \mathrm{s}$ was about $15^{\circ} \mathrm{C}$ higher than that of CCSSECAC at an airflow speed of $1 \mathrm{~m} / \mathrm{s}$, even if the pressure drop was greater.

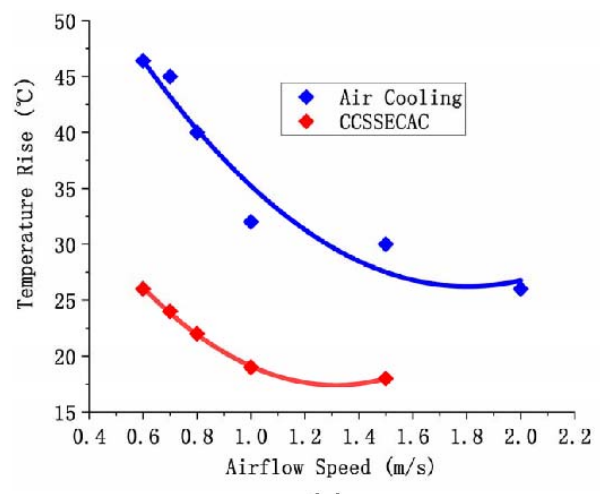

(a)

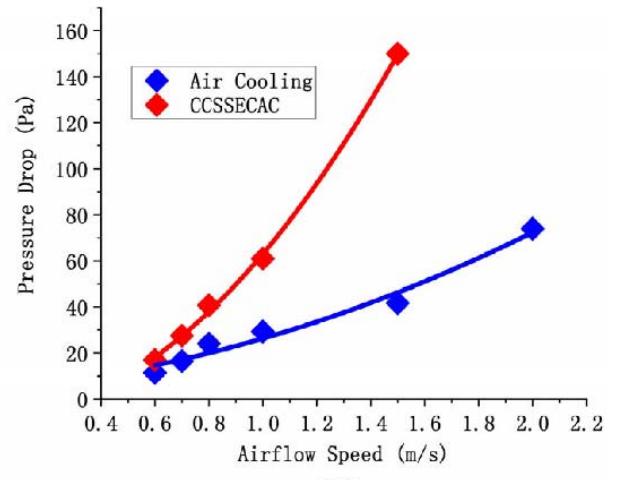

(b)

Figure 8. Comparison under the rated working conditions: (a) temperature rise at different airflow speeds and (b) airflow friction losses at different airflow speeds.

With the increase in airflow speed, the gap of the temperature rise between the two cooling systems tended to narrow. This was closely related to the physical properties of the evaporative cooling medium. Table 3 lists the boiling point at atmospheric pressure and the air temperature at the outlet of the stator model. The heat flux of the rated load was low. Even at the lowest airflow speed, the air temperature at the stator outlet was almost equal to the boiling point of the refrigerant. With the increase in airflow speed, the refrigerant was still in the state of supercooling when leaving the stator model. As a result, the phase transition was very inadequate or even impossible, which significantly weakened the effect of phase-change cooling.

Table 3. Temperature at the outlet of the stator model for CCSSECAC under the rated working conditions.

\begin{tabular}{ccccccc}
\hline Airflow Speed $(\mathrm{m} / \mathrm{s})$ & 0.6 & 0.7 & 0.8 & 1 & 1.5 & Boiling Point \\
\hline Temperature $\left({ }^{\circ} \mathrm{C}\right)$ & 46.7 & 46.2 & 44.6 & 43 & 42.7 & 46.7 \\
\hline
\end{tabular}

\subsection{Comparison of the Current Density with Equal Temperature Rise}

In this part, $50{ }^{\circ} \mathrm{C}$ was selected as the same temperature rise benchmark to compare the current density under different cooling conditions. The details of this comparison are given in Figure 9. At each airflow speed, CCSSECAC could achieve a current density that was at least $1 \mathrm{~A} / \mathrm{mm}^{2}$ higher than air cooling. Since the copper loss is proportional to the quadratic power of the current, this degree of difference in current density shows a very substantial improvement for air-cooling systems. This benefit was more prominent when the airflow speed did not exceed $1 \mathrm{~m} / \mathrm{s}$. When the airflow speed reached $1.5 \mathrm{~m} / \mathrm{s}$, 
the airflow friction loss of CCSSECAC was twice that of the air-cooling system, while the heat dissipation capacity of CCSSECAC was only $70 \%$ higher than that of the air-cooling system. It is worth mentioning that CCSSECAC increased the current densities far beyond those of the air-cooling system, comparable to hydrogen cooling, when the hottest spot on the coil was no more than $90^{\circ} \mathrm{C}$.

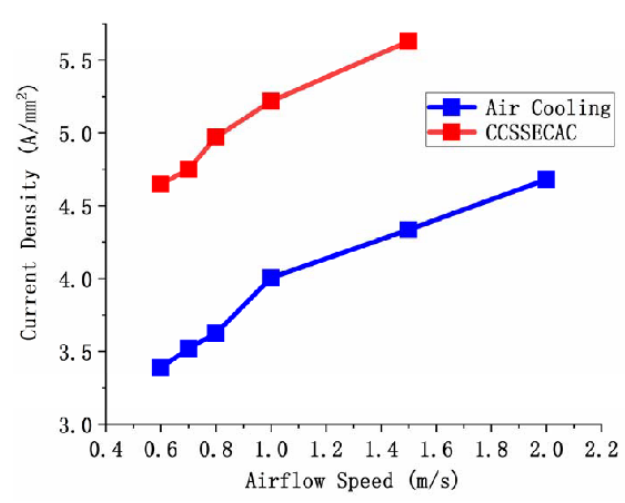

(a)

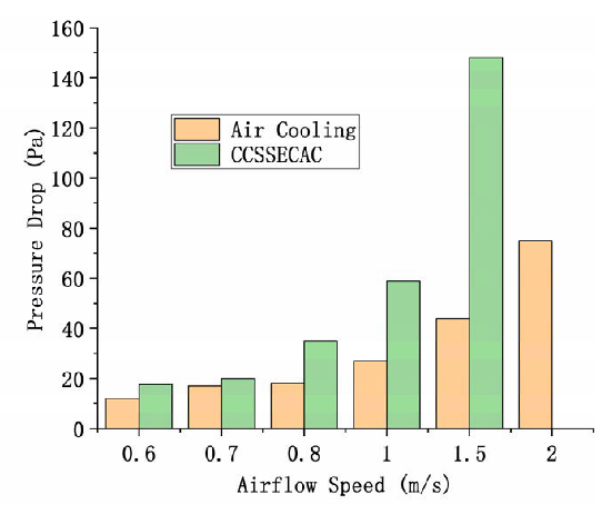

(b)

Figure 9. Comparison at a temperature rise of $50{ }^{\circ} \mathrm{C}$ : (a) current density at different airflow speeds and (b) airflow friction loss at different airflow speeds.

Table 4 shows once again the importance of the phase change in CCSSECAC. The superheating of the evaporative cooling medium at the stator outlet decreased with the increase in the airflow speed until it approached the boiling point. Perhaps at the airflow speed of $1.5 \mathrm{~m} / \mathrm{s}$, the droplets did not have time to absorb enough heat and completely evaporate when they went through the stator ducts.

Table 4. Temperature at the outlet of the stator model for CCSSECAC when the temperature rise was $50^{\circ} \mathrm{C}$.

\begin{tabular}{ccccccc}
\hline Airflow Speed $(\mathrm{m} / \mathrm{s})$ & 0.6 & 0.7 & 0.8 & 1 & 1.5 & Boiling Point \\
\hline Temperature $\left({ }^{\circ} \mathrm{C}\right)$ & 51.2 & 50.9 & 49 & 47.6 & 46.3 & 47.6 \\
\hline
\end{tabular}

\subsection{Comparison of the Airflow Friction Loss for the Same Airflow Speed}

The existing experience shows that the airflow friction loss under air-cooling conditions is not sensitive to the heat load of the motor, but is sensitive to the fluid density and velocity. In CCSSECAC, a certain number of refrigerant droplets were sprayed into the airflow and exchanged momentum with the airflow. In this case, the sensitivity of the airflow friction loss was not significantly changed due to the small mass flow rate of the coolant. It was just that the airflow friction loss of the mixed fluid was more sensitive to the flow rate than pure air.

Figure 10 compares the trends of the airflow friction loss with varying heating power under the air cooling and CCSSECAC conditions. Neither of the two figures reflects the trend of airflow loss rising obviously with the heat load. When the current density increased, the pressure drop that was generated in the stator ducts tended to decrease slightly under CCSSECAC conditions. This was possible because the increase in heat flux accelerated the droplet gasification such that the droplets gain a little bit of momentum from the expansion. 


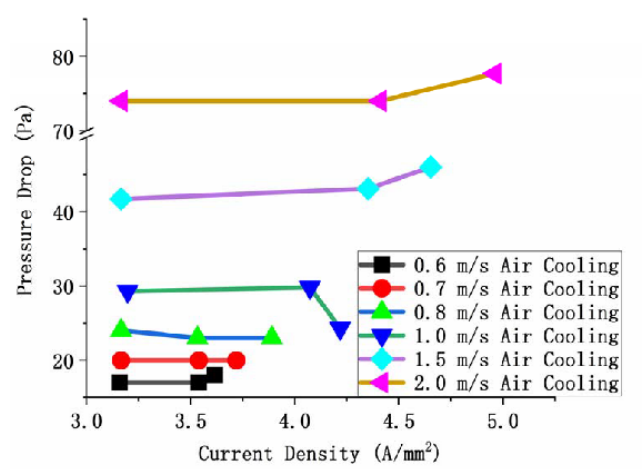

(a)

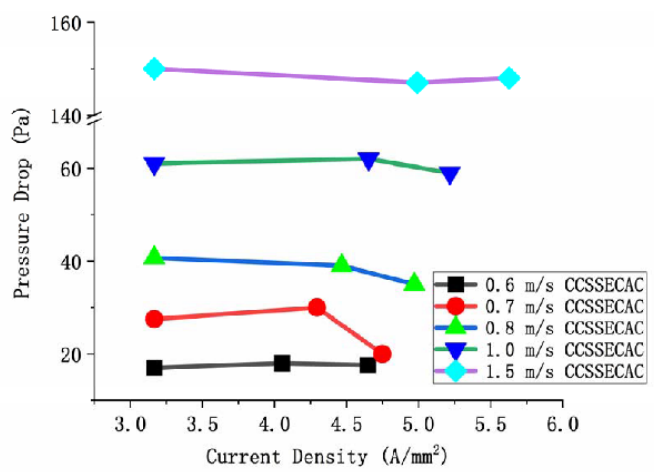

(b)

Figure 10. Airflow friction loss was not relevant to the heat load: (a) airflow friction loss in the air-cooling state and (b) airflow friction loss in the CCSSECAC state.

\section{Discussion}

Due to the introduction of volatile substances into the motor, the gas properties in the CCSSECAC were changed. In the air-cooling state, only pure air participated in the heat exchange cycle. In CCSSECAC, the heat transfer gas was a mixture of air and refrigerant vapor.

The calculation of the gas composition should start from the condenser outlet because at the outlet of the condenser, the refrigerant vapor reaches saturation. After measuring the outlet temperature of the condenser, the refrigerant saturated vapor pressure at the current temperature can be obtained. The ratio of refrigerant saturated vapor pressure to condenser outlet absolute pressure is the mole fraction of refrigerant vapor:

$$
\chi_{1}=\frac{P_{\text {sat }}\left(T_{0}\right)}{P_{0}}
$$

where $\chi_{1}$ is the mole fraction of refrigerant vapor, $T_{0}$ is the temperature at the condenser outlet, $P_{\text {sat }}\left(T_{0}\right)$ is the refrigerant saturated vapor pressure and $P_{0}$ is the absolute pressure at the condenser outlet.

The density and specific heat capacity of the mixture can be calculated as follows:

$$
\begin{gathered}
\rho_{\text {mix }}=\chi_{1} \rho_{1}+\left(1-\chi_{1}\right) \rho_{\text {Air }} \\
C_{p, \text { mix }}=\chi_{1} C_{p, 1}+\left(1-\chi_{1}\right) C_{p, \text { Air }}
\end{gathered}
$$

where $\rho_{\text {mix }}$ and $C_{p, \text { mix }}$ are the average density and specific heat at a constant pressure of the mixer, respectively; $\rho_{1}$ and $C_{p, 1}$ are the density and specific heat capacity at a constant pressure of refrigerant vapor, respectively; $\rho_{A i r}$ and $C_{p, A i r}$ are the density and specific heat capacity at a constant pressure of air, respectively.

To raise the temperature of air and mixture at the same flow rate, the power to be absorbed or released is

$$
W=C_{p, \operatorname{mix}} \rho_{\text {mix }} U S \cdot \Delta T
$$

where $W$ is the power of the heater or cooler, $U$ is the average velocity of the mixer and $S$ is the flow area.

For example, the physical properties of air mixed with a saturated vapor of R113 at $25^{\circ} \mathrm{C}$ and $1 \mathrm{~atm}$ are shown in Table 5. If the temperature and gage pressure at the condenser outlet are $25^{\circ} \mathrm{C}$ and $0 \mathrm{~atm}$ respectively, the density of the mixture will rise to 2.2 times that of air when we choose R113 as the coolant. The large density is consistent with the characteristics of the airflow friction loss of CCSSECAC. There is also good news in the table. According to Equation (7), the flow rate of mixed gas can be reduced by half under the condition of constant temperature rise, which is only due to the convective heat transfer of the mixture. Considering the huge latent heat of droplet evaporation, the strong cooling capacity of CCSSECAC in the experiments is self-evident. 
Table 5. Physical properties of air mixed with saturated R113 vapor at $25^{\circ} \mathrm{C}$ and $1 \mathrm{~atm}$ (absolute pressure).

\begin{tabular}{cccc}
\hline Property & Air & R113 (Vapor) & Mixture \\
\hline Density $\left(\mathrm{Kg} / \mathrm{m}^{3}\right)$ & 1.005 & 0.6593 & 2.255 \\
\hline Specific Heat Capacity $(\mathrm{J} /(\mathrm{kg} \cdot \mathrm{K}))$ & 1.293 & 3.4672 & 0.852 \\
\hline$C_{p} \cdot \rho\left(\mathrm{J} /\left(\mathrm{m}^{3} \cdot \mathrm{K}\right)\right)$ & 1.2995 & 2.2859 & 1.9213 \\
\hline Saturated Vapor Pressure $(\mathrm{atm})$ & - & 0.44245 & - \\
\hline
\end{tabular}

The thin-film heater could not load more heat due to the cooling capacity of CCSSECAC being much better than expected. However, according to the existing experimental results, under the condition of a low flow rate and high current density, CCSSECAC still had a large margin to bear a higher thermal load. For a motor with class $\mathrm{F}$ insulation, a temperature rise of $50{ }^{\circ} \mathrm{C}$ does not fully utilize the manufacturing material.

\section{Conclusions}

In this study, the principle of CCSSECAC was analyzed and an improved configuration with an ultrasonic atomization device was proposed. The effectiveness of the ultrasonic atomizer is verified by experiment. The CCSSECAC with the ultrasonic atomizer had almost twice the cooling capacity of the air-cooled system. Furthermore, without the complexity of the pressurization system for spray nozzles, ultrasonic atomizers are easy to install and control.

Compared with CCSSECAC using a nozzle, CCSSECAC using an ultrasonic atomizer urgently needs to find ultrasonic vibrators that can support a large flow. An ultrasonic atomizer is driven by a resonant circuit, and its electromagnetic compatibility needs further study.

Through this investigation, it was found that the advantage of CCSSECAC with an ultrasonic atomizer was strong heat transfer capacity, while the disadvantage was that the airflow friction loss increased too fast with the flow rate. Nevertheless, it was still a feasible solution in the application scenarios where water and hydrogen cooling devices were limited.

Author Contributions: Conceptualization, Y.W.; methodology, Y.W.; investigation, Y.W.; writingoriginal draft preparation, Y.W.; supervision, L.R.; project administration, L.R. Both authors have read and agreed to the published version of the manuscript.

Funding: This research was funded by Dongfang Electric Machinery Co., Ltd.

Institutional Review Board Statement: Not applicable.

Informed Consent Statement: Not applicable.

Data Availability Statement: The study did not report any data.

Conflicts of Interest: The authors declare no conflict of interest.

\section{References}

1. Ghahfarokhi, P.S.; Podgornovs, A.; Kallaste, A. Oil Spray Cooling with Hairpin Windings in High-performance Electric Vehicle Motors 2021. In Proceedings of the 2021 28th International Workshop on Electric Drives: Improving Reliability of Electric Drives (IWED), Moscow, Russia, 27-29 January 2021; Volume 27, pp. 1-5.

2. Mudawar, I.; Bharathan, D.; Kelly, K. Two-phase Spray Cooling of Hybrid Vehicle Electronics. IEEE Trans. Compon. Packag. Technol. 2009, 32, 501-512. [CrossRef]

3. Popescu, M.; Staton, D.; Boglietti, A. Modern Heat Extraction Systems for Electrical Machines-A Review. In Proceedings of the 2015 IEEE Workshop on Electrical Machines Design, Control and Diagnosis (WEMDCD 2015), Turin, Italy, 26-27 March 2015; pp. 289-296.

4. Liu, C.; Xu, Z.; Gerada, D. Experimental Investigation on Oil Spray Cooling with Hairpin Windings. IEEE Trans. Ind. Electron. 2019, 67, 7343-7353. [CrossRef] 
5. Li, Z.; Fu, D.; Guo, J. Study on Spraying Evaporative Cooling Technology for the Large Electrical Machine. In Proceedings of the 2009 International Conference on Electrical Machines and Systems, Tokyo, Japan, 15-18 November 2009; Volume 15, pp. 1-4.

6. Yu, T.; Liu, Y.; Chen, L. Experimental Study on Insulation Structure of Medium Voltage Freon Resistant Motor for Ship. In Proceedings of the 2019 22nd International Conference on Electrical Machines and Systems, Harbin, China, 11-14 August 2019; Volume 11, pp. 1-4.

7. Zhenguo, L.; Shaoyan, L.; Lin, R. The Experimental Studies on Spray Parameters in Spray Evaporative Cooling Motors. In Proceedings of the 2017 20th International Conference on Electrical Machines and Systems (ICEMS), Sydney, Australia, 11-14 August 2017; Volume 11, pp. 1-4.

8. Zhang, H.; Zhang, X.; Yi, X.L. Effect of Wettability on Droplet Impact: Spreading and Splashing. Exp. Therm. Fluid Sci. 2021, 124, 110369. [CrossRef]

9. Tian, J.; Chen, B. Dynamic Behavior of Non-evaporative Droplet Impact on a Solid Surface: Comparative Study of R113, Water, Ethanol and Acetone. Exp. Therm. Fluid Sci. 2019, 105, 153-164. [CrossRef]

10. Zang, D.Y.; Tarafdar, S.; Tarasevich, Y.Y. Evaporation of a Droplet: From Physics to Applications. Phys. Rep. 2019, 804, 1-56. [CrossRef]

11. Sazhin, S.S.; Rybdylova, O.; Crua, C. A Simple Model for Puffing/micro-explosions in Water-fuel Emulsion Droplets. Int. J. Heat Mass Transf. 2019, 131, 815-821. [CrossRef]

12. Volkov, R.S.; Strizhak, P.A. Research of Temperature Fields and Convection Velocities in Evaporating Water Droplets Using Planar Laser-induced Fluorescence and Particle Image Velocimetry. Exp. Therm. Fluid Sci. 2018, 97, 392-407. [CrossRef]

13. Strizhak, P.A.; Volkov, R.S.; Castanet, G. Heating and Evaporation of Suspended Water Droplets: Experimental Studies and Modelling. Int. J. Heat Mass Transf. 2018, 127, 92-106. [CrossRef]

14. Liang, G.; Mudawar, I. Review of Spray Cooling-Part 1: Single-phase and Nucleate Boiling Regimes, and Critical Heat Flux International Journal of Heat and Mass Transfer. Int. J. Heat Mass Transf. 2017, 115, 1174-1205. [CrossRef]

15. Dong, H.; Ruan, L.; Wang, Y.; Yang, J.; Liu, F.; Guo, S. Performance of air/spray cooling system for large-capacity and high-powerdensity motors. Appl. Therm. Eng. 2021, 192, 116925. [CrossRef]

16. Lang, R.J. Ultrasonic Atomization of Liquids. Acoust. Soc. Am. J. 1962, 34, 6. [CrossRef]

17. Avvaru, B.; Patil, M.N.; Gogate, P.R. Ultrasonic Atomization: Effect of Liquid Phase Properties. Ultrasonics 2006, 44, 146-158. [CrossRef] [PubMed]

18. Zhang, Y.; Yuan, S.; Wang, L. Investigation of Capillary Wave, Cavitation and Droplet Diameter Distribution During Ultrasonic Atomization. Exp. Therm. Fluid Sci. 2021, 120, 110219. [CrossRef]

19. Haoyu, D.; Haiwang, L.; Zhi, T.; Ruquan, Y. Study on Particle Size Control of Tracer Particles under Rotating Channel Conditions. J. Eng. Thermophys. 2019, 40, 2353-2358.

20. Minghu, Y.; Yuqiu, Z.; Zhengzhong, Q.; Bo, Z.; Junhong, Z. Research on Loss Separation Method of Permanent Magnet Snchronous Motor. Small Spec. Electr. Mach. 2015, 43, 14-18. 